\title{
An assessment of dermal exposure to semi-synthetic metal working fluids by different methods to group workers for an epidemiological study on dermatitis
}

\author{
B van Wendel de Joode, E P B Bierman, D H Brouwer, J Spithoven, H Kromhout
}

Occup Environ Med 2005;62:633-641. doi: 10.1136/oem.2004.015396

See end of article for authors' affiliations

.....................

Correspondence to: Dr H Kromhout

Environmental and

Occupational Health

Division, Institute for Risk

Assessment Sciences,

Utrecht University, PO Box 80176, 3508 TD Utrecht, Netherlands; h.kromhout@ iras.uu.nl

Accepted

25 February 2005
Background: Assessment of exposure assessment to metal working fluids (MWF) has almost exclusively focused on inhalation exposure.

Aims: To assess levels of, and factors affecting, dermal and inhalation exposure to semi-synthetic MWF, and to identify suitable dermal exposure grouping schemes among metal workers for an epidemiological survey on dermatitis of hands, forearms, and head.

Methods: A cross-sectional survey was conducted in four metal working machining departments of a truck manufacturing plant, estimating dermal and inhalation exposure levels to semi-synthetic metal working fluids (SMWF) in machine operators. Dermal exposure levels to SMWF were estimated by three different methods for dermal exposure assessment (VITAE, surrogate skin pad method, and a semi-quantitative dermal exposure assessment method (DREAM)).

Results: The identified factors affecting dermal exposure were similar for the three methods, although differences were found for estimated variability in dermal exposure levels between groups, within groups (among workers), and from day to day. With the VITAE method differences in exposure levels were detected between workers that were not detected with the surrogate skin pad method, and only partly with the DREAM method.

Conclusions: Considering the additional effort and costs that use of the VITAE method entailed, the observational semi-quantitative DREAM method appeared to be more efficient for grouping of dermal exposure levels for the epidemiological study on dermatitis.
M etal working fluids (MWF) is the general term given to a range of products used as lubricants and coolants during the machining or treatment of metal components. ${ }^{1}$ MWF are normally categorised as straight oil, soluble oil, semi-synthetic, and synthetic metal working fluids, of which the last three groups are designed to be diluted with water. ${ }^{2}$

Both straight oil and water based MWF have been associated with an increased risk of several digestive cancers, prostate cancers, and leukaemia..$^{3-5}$ In addition, MWF may cause respiratory health effects and dermatitis. ${ }^{6-10}$

During machining operations, MWF exposures can occur by both inhalation, resulting from breathing MWF mist or aerosols, and skin contact, for example resulting from fluid splashing onto the worker from the machine or when the worker is handling contaminated surfaces. ${ }^{2}$ Exposure assessment studies have almost exclusively focused on airborne exposure. ${ }^{11-14}$ To our knowledge, only three studies quantified dermal exposures. ${ }^{15-17}$

As dermal exposure levels generally do not correlate with inhalation exposure levels, ${ }^{18}$ it is useful to quantify dermal exposure in MWF exposed workers for epidemiological studies on local effects such as dermatitis, and systemic effects such as cancer, as MWF may penetrate the skin and contribute to internal exposure levels of MWF. Dermal exposure can be measured by different methods, including surrogate skin methods (for example, skin pads) ${ }^{19}$ and fluorescent tracer methods. ${ }^{20}$ Dermal exposure can also be assessed semi-quantitatively, by using DREAM, a recently developed observational generic dermal exposure assessment method. ${ }^{21-23}$

The aims of this study were: (1) to assess levels of, and factors affecting, dermal and inhalation exposure to semi-synthetic metal working fluids (SMWF); and (2) to identify suitable dermal exposure grouping schemes among metal workers for an epidemiological survey on dermatitis of hands, forearms, and head.

\section{METHODS}

Study design and workplace characteristics

This study was performed to characterise exposure levels to SMWF of 80 metal workers, who participated in a crosssectional, exploratory, epidemiological survey on dermatitis. The epidemiological study on dermatitis was described in detail elsewhere. ${ }^{24}$

We conducted a cross-sectional exposure assessment study in four metal working machining departments of a truck manufacturing plant, estimating dermal and inhalation exposure levels to SMWF in 36 of the 80 machine operators. Machinery varied from open, manually controlled machines, to closed, computer numerically controlled machines that all required manual changing of work pieces. Workers' major activities were: putting in, and taking out, work pieces, cleaning of work pieces with compressed air, and tuning machines. Each worker operated a group of metal working machines during a working day. In total 27 groups of machines were present at the four departments.

Table 1 describes factors that may affect exposure. The 27 groups of machines were regrouped into four types of machines on the basis of machine characteristics such as

Abbreviations: DREAM, dermal exposure assessment method; $G M$, geometric mean; LOD, limit of detection; MWF, metal working fluids; SMWF, semi-synthetic metal working fluids; VITAE, video imaging technique for assessing dermal exposure 
Table 1 Frequency of potential factors affecting exposure for each department

\begin{tabular}{|c|c|c|c|c|}
\hline \multirow[b]{2}{*}{ Determinant } & \multicolumn{4}{|c|}{ Department } \\
\hline & 1 & 2 & 3 & 4 \\
\hline \multicolumn{5}{|c|}{ Type of machines operated by worker } \\
\hline Open & $4(31 \%)$ & $2(11 \%)$ & $0(0 \%)$ & $0(0 \%)$ \\
\hline Closed I* & $9(69 \%)$ & $9(50 \%)$ & 7 (100\%) & $3(23 \%)$ \\
\hline Closed II† & $0(0 \%)$ & $7(39 \%)$ & $0(0 \%)$ & $0(0 \%)$ \\
\hline Closed III & $0(0 \%)$ & $0(0 \%)$ & $0(0 \%)$ & $10(77 \%)$ \\
\hline \multicolumn{5}{|c|}{ No. of work pieces handled by worker } \\
\hline $0-53$ & $2(15 \%)$ & $1(5 \%)$ & $4(57 \%)$ & $7(54 \%)$ \\
\hline$\geqslant 53-200$ & $10(77 \%)$ & $5(28 \%)$ & $3(43 \%)$ & $6(46 \%)$ \\
\hline$\geqslant 200$ & $1(8 \%)$ & $12(67 \%)$ & $0(0 \%)$ & $0(0 \%)$ \\
\hline \multicolumn{5}{|l|}{ Worker's overall } \\
\hline Short sleeved & $6(46 \%)$ & $13(72 \%)$ & $4(57 \%)$ & $8(62 \%)$ \\
\hline Long sleeved & $7(54 \%)$ & $5(28 \%)$ & $3(43 \%)$ & $5(38 \%)$ \\
\hline \multicolumn{5}{|c|}{$\begin{array}{l}\text { Worker used compressed air to } \\
\text { clean work pieces }\end{array}$} \\
\hline No & $2(15 \%)$ & $0(0 \%)$ & $0(0 \%)$ & $5(38 \%)$ \\
\hline Yes & $11(85 \%)$ & $18(100 \%)$ & 7 (100\%) & $8(62 \%)$ \\
\hline \multicolumn{5}{|c|}{ Worker used gloves } \\
\hline No & $2(15 \%)$ & $0(0 \%)$ & 1 (14\%) & $4(31 \%)$ \\
\hline Yes & $11(85 \%)$ & $18(100 \%)$ & $6(86 \%)$ & $9(69 \%)$ \\
\hline \multicolumn{5}{|c|}{$\begin{array}{l}\text { *Closed I: machine is closed during metal working. } \\
\text { †Closed II: machine is closed during metal working, mould automatically moves outside metal working machine } \\
\text { after finishing. } \\
\text { †Closed III: machine is closed during metal working, mould automatically moves outside metal working machine } \\
\text { after finishing, use of hoist. }\end{array}$} \\
\hline
\end{tabular}

open versus closed machines, type of mould, and whether a hoist is used when taking out work pieces. The number of work pieces handled was grouped into three categories on the basis of their 25th and 75th centiles.

\section{Measurement strategy}

We used a machine based measurement strategy as within each department, workers rotated between groups of metal working machines on a weekly basis, and exposure levels were likely to be principally determined by the type of metal working machine operated by the workers. Full-shift workers' dermal exposure levels to two types of SMWF, both containing more than $90 \%$ water and about $1 \%$ ethylene glycol were assessed for all groups of SMWF machines $(n=27)$ present at the four departments, of which 17 groups of machines were assessed repeatedly, resulting in 51 data points (see table 2 ).

Dermal exposure levels on hands, forearms, and head/ neck, were estimated by three different methods: two quantitative (surrogate skin, fluorescent tracer technique VITAE) and one semi-quantitative method (DREAM). At one of the departments (department 4) only two methods (surrogate skin, DREAM) could be applied, because its machinery was connected to a central metal working fluids reservoir of $20000 \mathrm{l}$, instead of independent reservoirs. We could not add fluorescent tracer to the central reservoir, as required by the VITAE method. In six cases, one of the three methods was missing due to organisational and technical problems. Exclusion of these observations (except for department 4) resulted in 51 data points, comprising data for 27 groups of machines and 36 workers. Full-shift personal air samples were obtained for 33 of 36 workers $(n=46)$, including 26 of the 27 machines. All workers gave written, informed consent.

\section{Dermal exposure assessment methods Video imaging technique for assessing dermal exposure (VITAE) method}

The VITAE method is based on detection of fluorescence on skin after adding a tracer, a fluorescent whitening agent, to the substance for which dermal exposure is assessed. ${ }^{25}{ }^{26} \mathrm{We}$ applied the method as described by Bierman and colleagues, ${ }^{27}$ who adapted the image analysis, and used Tinopal CBS-X (Ciba-Geigy) as a tracer to water soluble pesticides.

Prior to field measurements, a calibration curve was made to determine the relation between the amount of fluorescence and the amount of tracer on skin according to Bierman and colleagues. ${ }^{27}$ We made pre-images of hands and forearms of seven subjects with white $(n=3)$ and dark $(n=4)$ skin colours. Subsequently, we applied $10 \mu \mathrm{l}$ of five concentrations of Tinopal dissolved in water soluble metal working fluids, ranging from 0.04 to $0.4 \mathrm{~g} / \mathrm{l}$, on one to eight sites of hands and forearms of the subjects (total $n=157$ ), and made postimages of hands and forearms. Pre- and post-images were analysed using SCIL image, calculating average grey values of spots and average grey values of underlying skin, and average amount of ng tracer for each spot.

A mixed linear regression model (REML, compound symmetry covariance structure) was applied to determine the relation between amount of fluorescence and the amount of tracer on skin, including log transformed mean grey value of spots as explanatory variable, mean amount of tracer, and log transformed mean grey value of skin as fixed effects, and subject as random effect (equation 1). Residuals were normally distributed. The equation explained $83 \%$ of total variance.

Average amount of tracer (ng) $=-3.77+6.40 \times$ $\ln$ (average spot fluorescence) $-6.22 \times \ln$ (average skin fluorescence) (equation 1)

For each measured body segment the limit of detection (LOD) was set at $300 \mathrm{ng}$ as this was the lowest amount of tracer that could be visibly distinguished by two of the authors (BW, EB) who independently revised all images of the calibration curve. Samples below the limit of detection were set on $1 / \sqrt{2}$ of LOD. Image analysis of all segments was performed twice. The total coefficient of variation $\left(\mathrm{CV}_{t}\right)$ of image analysis was $14.4 \%$.

Tinopal CBS-X (Ciba-Geigy) was added to metal working machines to obtain a concentration of about $100 \mathrm{mg}$ Tinopal/l metal working fluid. Source samples of machines were taken during the measurements in order to determine the exact 
concentration of Tinopal in the metal working fluids. Samples were chemically analysed for Tinopal concentration using a C18-HPLC (Applied Biosystems 400 SDS), a UV detector at $343 \mathrm{~nm}$ (Applied Biosystems 759A variable UV detector), and a Phenomemex Luna $5 \mu \mathrm{m} \mathrm{C18} \mathrm{(2),} \mathrm{150/}$ $4,6 \mathrm{~mm}$ column, at a flow of $1.0 \mathrm{ml} / \mathrm{min}$, with ammonium acetate $(5 \mathrm{~g} / \mathrm{l})$ with $70 \%$ methanol as an eluent. The calibration curve was linear between 0.001 and $0.1 \mathrm{mg} / \mathrm{l}$. The average recovery was $96 \%(n=2,95.4 \%$ and $96.5 \%)$.

Dermal exposure measured by VITAE was expressed as ng tracer $/ \mathrm{cm}^{2}$ skin. Subsequently, we expressed dermal exposure as $\mathrm{mg}$ metal working fluid per $\mathrm{cm}^{2}$ skin as concentration of Tinopal in the metal working fluid (\%ww) was known. When a worker operated more than one metal working machine, we used the average concentration of Tinopal in the metal working fluids of the different machines.

\section{Surrogate skin}

Alpha-cellulose pads were placed between two circular Fixomul stretch bandage plasters (BDF), $5 \mathrm{~cm}$, with a circular opening on $2.5 \mathrm{~cm}$ in diameter in the upper plaster layer. The pads were located on hands (left or right hand side), wrists (left or right hand side), and neck. After measuring exposure, a Ø27 mm segment was punched out of the pad and transferred immediately in $1.5 \mathrm{ml}$ distilled water filled pre-coded, air closed PVC tubes. Tubes were vigorously shaken for 2 minutes and stored in a freezer $\left(-20^{\circ} \mathrm{C}\right)$ until they were analysed.

The SMWF for which dermal exposure was assessed, contained about $1 \%$ ethylene glycol (\%ww). Extraction took place by vortexing the tubes containing the filters and $1.5 \mathrm{ml}$ of distilled water for 30 seconds, shaking them in a horizontal position (Gerhard LS20) for 60 minutes, and vortexing again for 30 seconds. Chemical analysis consisted of the determination of the amount of ethylene glycol in the $1.5 \mathrm{ml}$ extraction liquid using a colorimetric assay, measuring reduction of light absorbance of potassium dichromate at $450 \mathrm{~nm}$ using a microtitre plate reader. The method is based on an assay designed in the 1950s for the determination of ethanol in blood ${ }^{28}$ and was modified to be able to detect ethylene glycol in metalworking fluids in the following manner. First, we used ethylene glycol instead of alcohol for the calibration curve; second, the chemical reaction directly took place in the distilled water in which the pads were extracted; third, after the chemical reaction has taken place, samples were centrifuged at $3000 \mathrm{~g}$ for 15 minutes in order to separate non-soluble components. Spike samples $(n=4)$, adding 5 and $15 \mu \mathrm{l}$ of metal working fluid to pads in triplicate, were taken to calculate the recovery of ethylene glycol for alpha-cellulose pads. The metal working fluid was taken from a metal working machine of the truck manufacturing plant. Average recovery was $86.3 \%$ (range 55$121 \%)$. As the range of recovery was rather large, all samples were analysed in duplicate, resulting in a total coefficient of variation $\left(\mathrm{CV}_{\mathrm{t}}\right)$ of $18.8 \%$.

On each measurement day one to three field blanks were taken (total $n=29$ ). LOD was calculated by average concentration measured on blanks summed by three times the standard deviation, resulting in an LOD of $49.1 \mathrm{mg}$ ethylene glycol per litre extraction liquid. For each machine that was operated by a studied worker a source sample was taken and the amount of ethylene glycol was chemically analysed. Subsequently, we expressed dermal exposure as $\mu \mathrm{g}$ metal working fluid per $\mathrm{cm}^{2}$ skin as concentration (\%ww) of ethylene glycol in the metal working fluid was known. When a worker operated more than one metal working machine, we used the average concentration of ethylene glycol in the metal working fluids of the different machines.

\section{DREAM}

The DREAM method is an observational semi-quantitative method to assess dermal exposures by systematically evaluating factors affecting exposure using pre-assigned default values. ${ }^{21}$ An occupational hygienist completed the DREAM questionnaire while observing during 10-30 minutes a worker whose dermal exposure was measured quantitatively at the same time.

The DREAM method is generic and designed for dermal exposure assessment in epidemiological and occupational hygiene surveys. The method comprises two parts: a multiple choice questionnaire, and an evaluation model. The questionnaire includes questions about: (1) the probability and intensity of the main dermal exposure routes: emission, deposition, and transfer for nine body parts; (2) information on clothing layer (that is, kind of material covering skin, replacement frequency of clothing, percentage of time gloves are being worn) for the worker performing the task; (3) physical and chemical characteristics of the substance to which dermal exposure occurs; and (4) percentage of working time a task is performed. Each answer of the questionnaire matches with an a priori assigned value. In the evaluation model of DREAM, the assigned values are used in an algorithm resulting in numerical estimates for exposure levels experienced by workers performing a certain task or job. For a detailed description of the method we refer to van Wendel de Joode and colleagues. ${ }^{21-23}$

\section{Personal air sampling}

Workers wore a Gilair pump (2 l/min) connected to a PAS-6 sampling head containing a Teflon filter $(37 \mathrm{~mm}$ diameter, $2 \mu \mathrm{m}$ pore size). Filters were analysed gravimetrically in order to determine total amounts of inhalable particles $\left(\mathrm{mg} / \mathrm{m}^{3}\right)$.

\section{Data analysis}

For reasons of comparison dermal exposure levels on hands, forearms, and head were multiplied by their body surface area. Exposure levels on hands and forearms were subsequently summed. Measurements covered approximately full shift exposure levels (median measurement time 411 minutes). Measurements below LOD were set on $1 / \sqrt{2}$ of LOD. Both dermal and inhalation exposure levels followed a log normal distribution. Statistical analyses were performed in SAS version 8.2 (SAS Institute, Cary, NC).

Potential exposure affecting factors (table 1) were first analysed univariately in a mixed linear regression analysis (REML, compound symmetric covariance structure) with log transformed dermal exposure values as dependent variable and "worker" as random effect. The estimated beta of an exposure affecting factor, included as a fixed effect, represents the average effect of this factor on the log transformed measured dermal and inhalation exposure levels of all workers (equation 2). The variable "worker" was included as a random effect to account for natural differences in workers' exposure levels (equation 2).

Subsequently, the factor was only included in the multivariate model if its $\mathrm{p}$ value was $<0.10$ in the univariate analysis for results of VITAE or pads. We verified whether the fixed effects included in the models showed interaction. Residuals of the mixed linear regression models were tested for normality and outliers. The residuals of the models approximated a normal distribution.

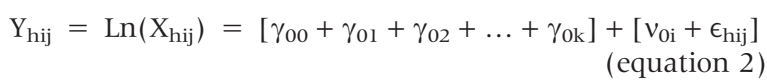

$\mathrm{Y}_{\mathrm{hij}}=$ the natural logarithm of the exposure concentration $\left(X_{\text {hij }}\right)$ measured for the $j$-th day of the $i$-th worker of the h-th department; 
Table 2 Geometric means (GMs) with geometric standard deviations (GSDs) in parentheses for total dermal exposure levels on hands and forearms and airborne exposure levels

\begin{tabular}{|c|c|c|c|c|c|c|c|c|c|c|}
\hline \multirow[b]{2}{*}{ Department } & \multirow[b]{2}{*}{$\mathrm{N} \S$} & \multirow[b]{2}{*}{ M† } & \multirow[b]{2}{*}{$W \ddagger$} & \multicolumn{3}{|l|}{ Dermal (mg) } & \multirow[b]{2}{*}{$\mathrm{N}$} & \multirow[b]{2}{*}{$M$} & \multirow[b]{2}{*}{ W } & \multirow[b]{2}{*}{ Airborne $\left(\mathrm{mg} / \mathrm{m}^{3}\right)$} \\
\hline & & & & VITAE & Pads & DREAM & & & & \\
\hline 1 & 13 & 5 & 9 & $2451(3.4)^{* *}$ & $5218(2.1)^{*}$ & 15901 (3.5) & 10 & 4 & 7 & $0.58(1.6)$ \\
\hline 2 & 18 & 11 & 12 & $1690(5.1)^{* *}$ & $3807(2.5)$ & 24737 (3.0)t† & 18 & 11 & 12 & $0.70(1.6)$ \\
\hline 3 & 7 & 4 & 6 & $254(6.2)$ & $2086(2.4)$ & $14826(3.1)$ & 7 & 4 & 6 & $0.66(1.6)$ \\
\hline 4 & 13 & 7 & 9 & N.A. & $3454(2.8)$ & $7096(3.6)$ & 11 & 7 & 8 & $0.55(1.6)$ \\
\hline Total & 51 & 27 & 36 & $1354(5.5)$ & $3706(2.5)$ & 14985 (3.5) & 46 & 26 & 33 & $0.63(1.6)$ \\
\hline
\end{tabular}

${ }^{*} \mathrm{p}<0.05,{ }^{* *} \mathrm{p}<0.01$ : GM larger than GM of department 3

$\uparrow p<0.10,+\uparrow p<0.05$ : GM larger than GM of department 4.

§Total number of samples.

†Number of groups of metal working machines.

$\ddagger$ Number of workers.

$\gamma_{00}=$ the true underlying mean of $\log$ transformed exposure averaged over all groups;

$\gamma_{01} \ldots \gamma_{0 \mathrm{k}}=$ fixed effects of $\mathrm{k}$ factors affecting exposure;

$v_{0 \mathrm{i}}=$ random effect of the $i$-th worker;

$\epsilon_{\text {hij }}=$ random error (within-worker; day-to-day variation).

When grouping the exposure levels of workers for an epidemiological study, the grouping ideally results in a maximum between-group variance and a minimum withingroup variance, because a relation between exposure and health effects is most likely to be identified for a maximum contrast in exposure. ${ }^{29}{ }^{30}$ Kromhout and Heederik ${ }^{30}$ defined this contrast in exposure as the ratio of the between-group variance $\left(S_{\mathrm{BG}}\right)$ and the sum of the between-group and within-group ( $\mathrm{SS}_{\mathrm{WG}}$ ) variance (equation 3 ).

$$
\mathrm{SS}_{\mathrm{BG}} /\left(\mathrm{SS}_{\mathrm{BG}}+\mathrm{SS}_{\mathrm{WG}}\right)
$$

(equation 3)

In order to evaluate different ways of grouping workers, dermal and inhalation exposure variance components were estimated for workers grouped according to "department" and "type of machine" operated. In addition, workers were grouped "determinant based", and by a combination of "department" and "type of machine operated". Exposure variance components could not be assessed between and within "groups of machines" as we had only few repeated measurements for the same workers operating a specific group of machines.

Variance components were estimated by PROC MIXED (restricted maximum likelihood (REML), compound symmetric covariance structure), including the grouping variable as a random effect with log transformed exposure levels as dependent variable. In order to distinguish within-group variance and day-to-day variance in addition to betweengroup variance, the models contained "worker" as a second random effect (equation 4).

$Y_{\text {hij }}=\operatorname{Ln}\left(X_{h i j}\right)=\left[\gamma_{00}\right]+\left[v_{0 g}+v_{1 i}+\epsilon_{\text {hij }}\right]$ (equation 4)

$\mathrm{Y}_{\mathrm{hij}}=$ the natural logarithm of the exposure concentration $\left(X_{\text {hij }}\right)$ measured for the $j$-th day of the $i$-th worker of the $h$-th department;

$\gamma_{00}=$ the true underlying mean of log transformed exposure averaged over all groups;

$v_{0 i}$ random effect of the $g$-th grouping variable;

$v_{1 \mathrm{i}}=$ random effect of the $i$-th worker;

$\epsilon_{\mathrm{hij}}=$ random error (within-worker; day-to-day variation).

Contrasts in exposure levels were subsequently estimated for the grouping variables ("department", "task",

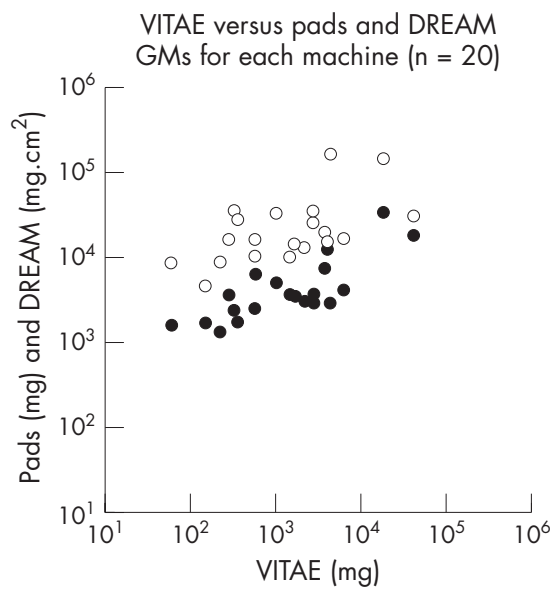

- VITAE - pads:
Spearman's $r=0.75(p<0.001)$
VITAE - DREAM:
Spearman's $r=0.57(p<0.01)$
Pads versus DREAM

GMs for each machine $(n=27)$

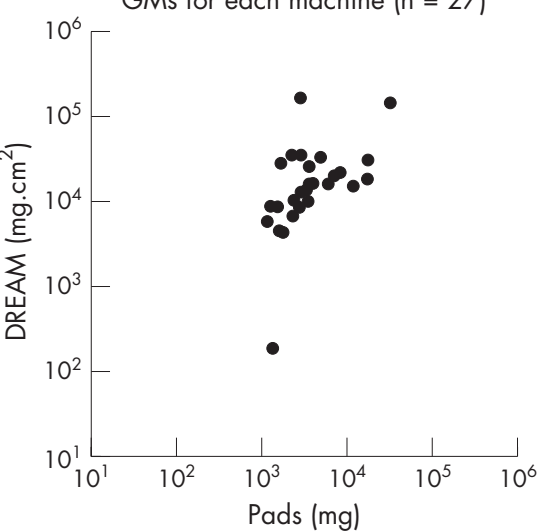

- Pads- DREAM:

Spearman's $r=0.59(p<0.01)$

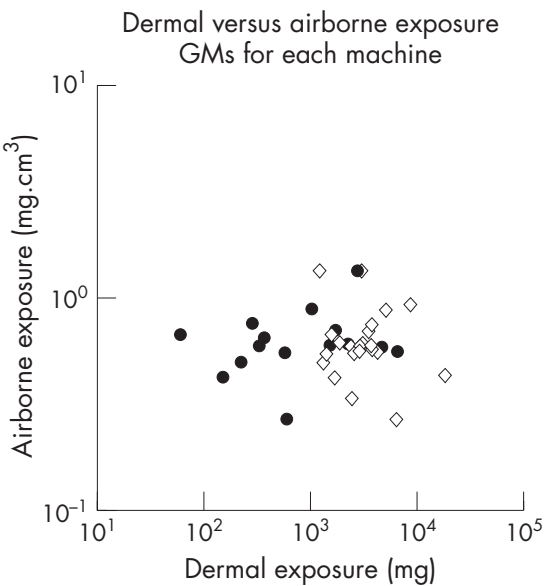

- VITAE - airborne exposure levels: Spearman's $r=0.38, p=0.11$ ( $\mathrm{n}=19$ )

$\diamond$ Pads - airborne exposure levels: Spearman's $r=0.27, p=0.18$ $(n=26)$

Figure 1 Geometric means (GMs) for each group of machines plotted for total dermal exposure levels on forearms and hands measured by VITAE, surrogate skin pads, and DREAM, and airborne exposure levels. 

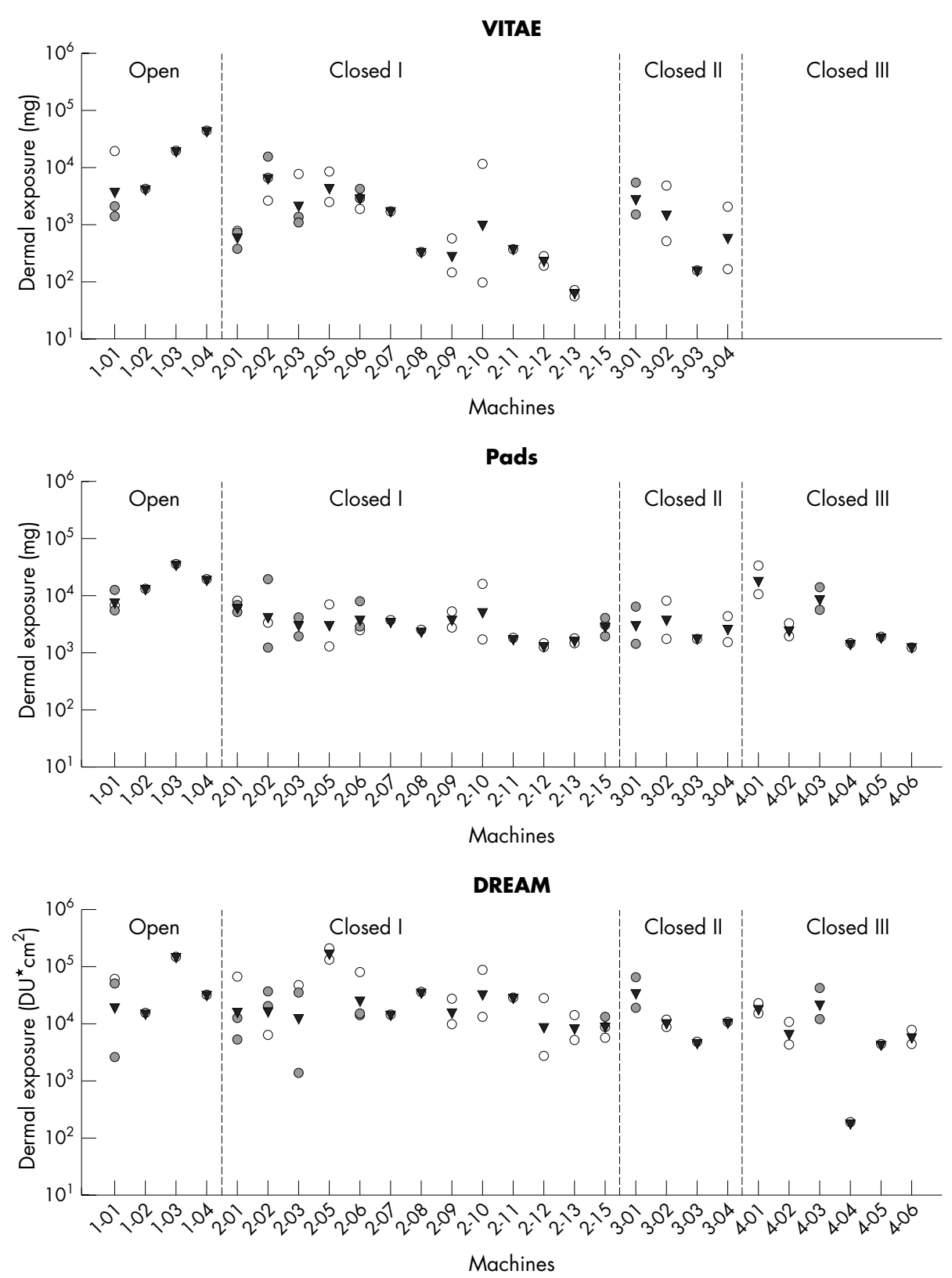

- Individual data point

- Individual data point of a worker repeatedly measured at the same machine

$\nabla$ Geometric mean

${ }^{\dagger} 1-01$ to $1-04=$ Open machines; $2-01$ to $2-15=$ Closed machines type I;

3-01 to 3-04 = Closed machines type II; 4-01 to 4-06 = Closed machines type III

Figure 2 Total dermal exposure levels of hands and forearms plotted for each group of machines, grouped by type of machinet, for VITAE, pads, and DREAM.

"machine", and combinations) by dividing the between group variance by the sum of the between group variance and the within group variance) (equation 3 ). ${ }^{29}$

\section{RESULTS}

All personal air samples had detectable exposure levels. Dermal exposure levels on hands and forearms were detectable for 35 of 38 samples (92\%) measured by the VITAE method, and for 33 of 51 samples $(65 \%)$ when measured by the pads. For exposure on face and neck, only 12 of 38 samples (32\%) showed detectable levels of SMWF for the VITAE technique, and only 1 of 6 samples (17\%) for the pads (results not shown). Detectable exposure levels on face and neck were most frequently found at department 2 ( 10 of $18(56 \%)>$ LOD) (results not shown). Due to the large amount of samples below LOD for exposure levels on face and neck, further data analyses were only performed for results of hands and forearms, and for personal air samples.

As can be seen from table 2, dermal exposure levels measured on pads were higher than exposure levels determined by the VITAE method. For both the VITAE method and surrogate skin pad method, geometric means 


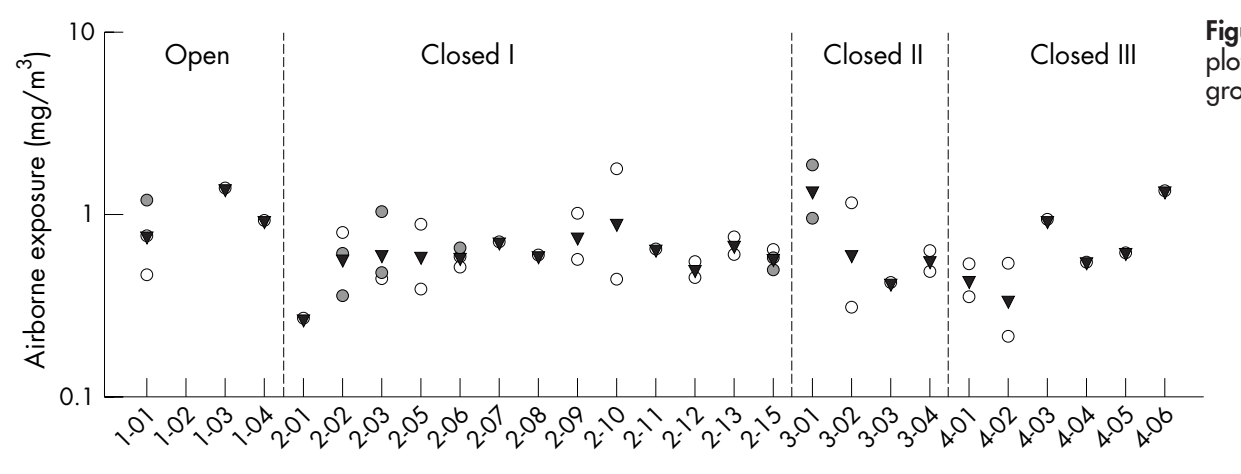

Figure 3 Airborne exposure levels plotted for each group of machine, grouped by type of machinet.

Machines

\begin{tabular}{ll|}
\hline & Individual data point \\
$\circ$ & Individual data point of a worker repeatedly measured at the same machine \\
$\nabla$ & Geometric mean \\
\hline
\end{tabular}

${ }^{\dagger} 1-01$ to $1-04=$ Open machines; $2-01$ to $2-15=$ Closed machines type $\mathrm{I}$;

3-01 to 3-04 = Closed machines type II; 4-01 to 4-06 = Closed machines type III

(GMs) of measured dermal exposure levels were highest for workers of department 1 , followed by workers of department 2. For the DREAM method, GMs were highest for department 2, followed by department 1 . Airborne exposure levels were similar throughout the departments with a relatively low variability $(\mathrm{GSD}=1.6)$ in exposure concentrations.

Geometric means (GMs) of dermal exposure levels on hands and fore arms for each group of machines measured by VITAE correlated well with dermal exposure levels measured with the pads (Spearman's $r=0.75$ ), and moderately with GMs assessed by DREAM (Spearman's $r=0.57$ ) (fig l). Also dermal exposure levels measured with the pads correlated moderately with the DREAM estimates $(\mathrm{r}=0.59, \mathrm{p}<0.01)$. Dermal exposure levels assessed by VITAE and pads did not correlate statistically significant with airborne exposure levels (Spearman's $\mathrm{r}=0.36(\mathrm{p}=0.11)$ and $\mathrm{r}=0.27 \quad(\mathrm{p}=0.18)$, respectively).

In fig 2 total dermal exposure levels of hands and forearms are plotted for each group of machines, grouped by type of machine for VITAE, pads, and DREAM. Variability in geometric mean exposure levels of groups of machines was larger for the VITAE method compared to pad and DREAM methods. Exposure levels differed especially between open and closed machines for dermal exposure levels measured by the VITAE method (see also table 3), but also within type of machines (fig 2). For airborne exposure levels no obvious differences between departments or between machines were present (fig 3).

Table 3 shows the relation between factors affecting exposure and log transformed measured dermal exposure levels as estimated with three dermal exposure assessment methods. As no differences in dermal exposure levels measured by VITAE or pads were found for different type of closed machines (type I, II, III) in the univariate mixed linear regression analyses, the type of machine the worker operated was entered as a binominal variable (open/closed machines) in the multiple regression model. As the univariate analysis showed that an increase in exposure level was only related to the upper category $(\geqslant 200$ pieces handled), the number of work pieces handled was also entered in the model as a binominal variable $(<200$ versus $\geqslant 200$ pieces handled).

From table 3 it becomes clear that three factors affected dermal exposure levels: "operating an open machine", "no gloves used", and the interaction term of ">200 work pieces handled" and "cleaning of work pieces with compressed air", showed a statistically significant $(\mathrm{p}<0.10)$ relation with $\log$ transformed dermal exposure levels measured by the VITAE method and pads. Two out of three factors showed also a statistically significant $(p<0.10)$ relation with log transformed dermal exposure levels as estimated by the semiquantitative DREAM method.

The percentage of total variability in dermal exposure levels explained by the three factors was comparable for VITAE and pads ( $42 \%$ and $43 \%$, respectively), and somewhat lower for DREAM (27\%). The identified factors affecting dermal exposure were related to departments, because "open" machines were only present at departments 1 and 2, and the combination of ">200 work pieces handled by worker" and "cleaning of work pieces with compressed air" also occurred only at departments 1 and 2. Workers not using gloves were seen at departments 1 and 3 , as well as 4 (table 1).

None of the factors showed a statistically significant $(\mathrm{p}<0.10)$ relation with log transformed airborne exposure levels.

Table 4 shows that, in general, variance components were largest for the VITAE method. The surrogate skin pad method did not show any within-group (between worker) variance, whereas the DREAM method did show a small within-group variance component and a relatively large day-to-day variance component. Within methods, different grouping schemes resulted in somewhat different estimates of between- and within-group variance components, but overall results were similar. For VITAE and DREAM, between-group variance was highest for the determinant based grouping (departments 1 and 2 versus departments 3 and 4), whereas for pads grouping according to "type of machine" resulted in the highest between-group variance. Airborne exposure levels did not show any between group variance.

\section{DISCUSSION}

In this study we estimated dermal exposure levels to SMWF by applying three different methods for dermal exposure assessment. The identified factors affecting dermal exposure were quite similar for the three methods, although differences were found with regard to variability in dermal exposure levels between groups, within groups among workers, and from day-to-day. For airborne exposure levels no exposure affecting factors could be identified and exposure levels varied almost exclusively from day-to-day. 
Table 3 Factors affecting exposure related to log transformed total dermal exposure levels on hands and forearms $(\mathrm{ln}(\mathrm{mg}))$ as assessed by three different methods

\begin{tabular}{|c|c|c|c|c|}
\hline \multirow[b]{2}{*}{ Determinant } & \multirow[b]{2}{*}{$\mathrm{N} \S$} & \multicolumn{3}{|l|}{ Method } \\
\hline & & $\begin{array}{l}\text { VITAE } \\
\mathrm{N} \dagger=38, \mathrm{~K} \ddagger=27\end{array}$ & $\begin{array}{l}\text { Pads } \\
N=51, K=36\end{array}$ & $\begin{array}{l}\text { DREAM } \\
\mathrm{N}=51, \mathrm{~K}=36\end{array}$ \\
\hline Intercept & & $5.4(1.0)^{* * * \star}$ & $7.8(0.3)^{* \star * *}$ & $8.6(0.5)^{\star * * *}$ \\
\hline Open machine & 6 & $2.9(0.6)^{* *}$ & $1.4(0.3)^{\star *}$ & $0.8(0.5)$ \\
\hline$>200$ work pieces handled & 13 & $-2.9(1.9)$ & $-1.3(0.8)$ & $-1.2(1.3)$ \\
\hline Cleaning work pieces with compressed air & 44 & $0.8(0.9)$ & $0.0(0.3)$ & $0.5(0.5)$ \\
\hline No gloves used & 7 & $2.9(1.0)^{*}$ & $1.5(0.3)^{* *}$ & $1.5(0.5)^{*}$ \\
\hline $\begin{array}{l}>200 \text { work pieces handled and cleaning } \\
\text { work pieces with compressed air }\end{array}$ & 4 & $3.9(2.0) \#$ & $1.7(0.9) \#$ & $2.6(1.3) \#$ \\
\hline \multirow{2}{*}{\multicolumn{2}{|c|}{$\%$ of total variance explained by fixed effects }} & $42 \%$ & $43 \%$ & $27 \%$ \\
\hline & & \multicolumn{3}{|c|}{$\begin{array}{l}\text { Random effects (random effects of model with random } \\
\text { effects only) }\end{array}$} \\
\hline $\mathrm{SS}_{\mathrm{BW}}$ & & $1.32(2.19)$ & $0.00(0.00)$ & $0.00(0.13)$ \\
\hline SSwwt† & & $0.49(0.93)$ & $0.48(0.84)$ & $1.15(1.44)$ \\
\hline \multicolumn{5}{|c|}{ 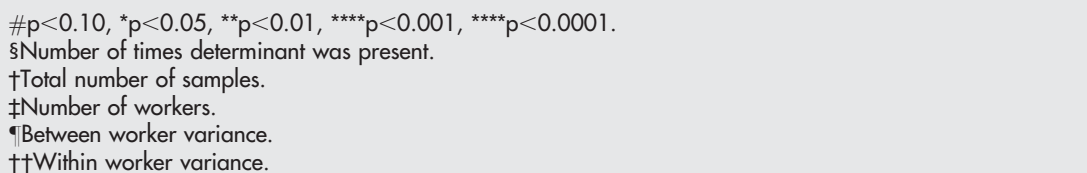 } \\
\hline
\end{tabular}

The lack of variability in airborne exposure levels between groups and within groups among workers can be explained because all four departments were situated in one large hall. The dermal exposure levels showed more variability than airborne exposure levels because dermal exposure seemed to be more directly related to machinery characteristics. Dermal exposure seemed to occur principally through splashes when taking out metal parts, by contact with metal parts contaminated with SMWF, and, to a lesser extent, through deposition of airborne particles.

Roff and colleagues ${ }^{17}$ measured skin exposure to MWF on hands using cotton measurement gloves $(n=7$ from one subject) and reported a geometric mean dermal exposure level of $2378 \mathrm{mg} / \mathrm{h}$ for hands. This is about a factor 4 and 12 higher than geometric mean dermal exposure levels measured in our study for pads $(541 \mathrm{mg} / \mathrm{h})$ and VITAE

Table 4 Estimated variance components for grouping according to department and type of machine with WORKER added as a second random effect, for log transformed total dermal exposure levels on hands and forearms, and log transformed airborne exposure levels

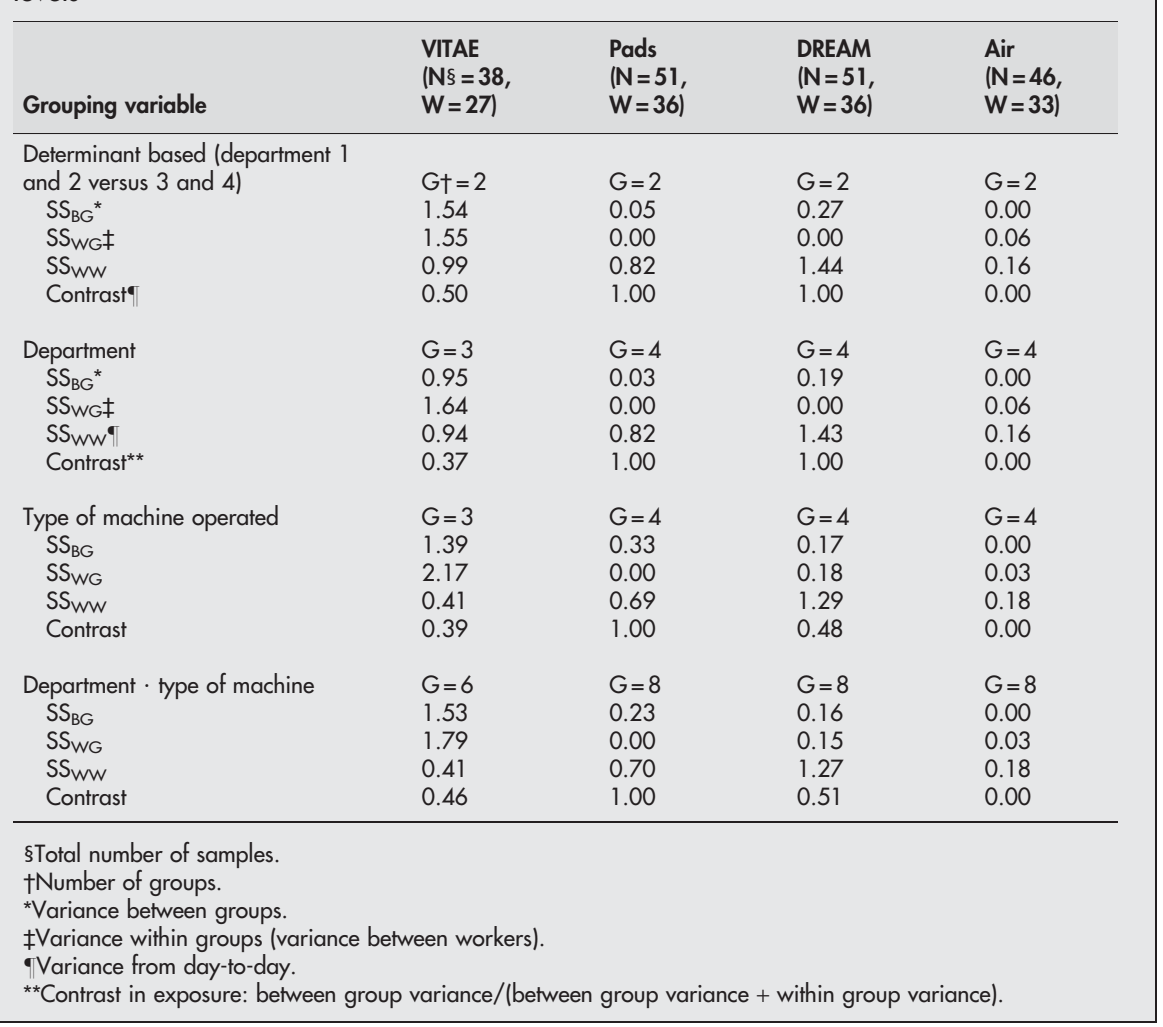


( $198 \mathrm{mg} / \mathrm{h}$ ). The differences may be partly explained due the use of different measurement techniques. Surrogate skin sampling techniques (pads, gloves), are likely to overestimate dermal exposure to metal working fluids, because the sampling material generally tends to capture and retain more contaminant than is attached to the skin. ${ }^{19}$ Cotton gloves are likely to absorb more metal working fluids than alphacellulose pads. On the other hand, median dermal exposure levels estimated by Roff and colleagues ${ }^{17}$ may have been higher as only during 14\% of the measurements the worker used gloves, whereas in our study during $86 \%$ of the measurements workers used gloves. In the current study, we found lower average dermal exposure levels, a factor 4 (pads) and 20 (VITAE), for workers wearing gloves.

Wassenius and colleagues ${ }^{15}$ developed a subjective semiquantitative observational method for the assessment of skin wetness. The authors found that a shorter cycle time was related to increased skin wetness. We found an association between the numbers of work pieces handled with dermal exposure for workers using compressed air to clean work pieces, which is in congruence with the relation found by Wassenius and colleagues ${ }^{15}$ for cycle time, as machines with shorter cycle times generally result in a higher number of work pieces handled by a worker. In addition, we found different dermal exposure levels for open and closed machines, but not for different types of closed machines, whereas Wassenius and colleagues ${ }^{15}$ did not find any relation between type of machine and skin wetness. Possibly, machinery studied by Wassenius and colleagues ${ }^{15}$ did not include open machines, or differences between open and closed machines are less adequately detected by observational methods. The latter can also be seen from our study because, as compared to the quantitative dermal exposure assessment methods, the DREAM method detected a smaller, not statistically significant, difference in exposure levels between open and closed machines. The three dermal exposure assessment methods differed with regard to estimated variability in exposure levels between groups, within groups among workers, and within workers from day-to-day. The VITAE method appeared to be the most sensitive method, as it detected differences in exposure levels between workers that were not detected by the surrogate skin pad method, and only partly by the DREAM method. Also, the VITAE method resulted in more detectable samples than the surrogate skin pad method.

The VITAE method is likely to provide a better estimate of "true" dermal exposure than the surrogate skin method. This is because the VITAE method covered exposure of both sides of both hands and forearms, while the surrogate skin method measured only exposure of a small surface area of the palmside of wrist, and back of hand. The surrogate skin pad method is likely to overestimate temporal (day-to-day) variability of dermal exposure, due to non-uniform distribution of exposure for a specific body location in combination with the relatively small surface area of the pad. ${ }^{31}$ The DREAM method showed the largest day-to-day variability, which may be explained by the short observation time of the method (10-30 minutes) from which a full-shift estimate was extrapolated. At least in one case, the DREAM observation was not representative for the full-shift exposure as the machines were malfunctioning when the worker was observed. This resulted in a very low DREAM estimate as for that moment no exposure occurred. In the rest of the cases, the extrapolation is likely to have introduced some random measurement error as well. Although the VITAE method seemed to provide more accurate dermal exposure estimates, the method's application is inhibited due to the dependence of a tracer that has to be added to the agent of interest. At one department measurements by VITAE were impossible because for practical reasons the tracer could not be added to the central SMWF reservoir.

Interestingly, the VITAE method and the surrogate skin pad technique identified the same factors affecting dermal exposure. The semi-quantitative DREAM method identified two of the three factors as well, and showed a smaller difference in dermal exposure for the third determinant.

With respect to grouping workers for our epidemiological study on dermatitis, we prefer the determinant based grouping of dermal exposure levels (departments 1 and 2, versus 3 and 4 ) as for the VITAE technique as well as the DREAM method contrasts in dermal exposures were largest for this way of grouping workers. The variability in dermal exposure levels between and within groups estimated with the surrogate skin pad method were not very informative, as the pad method did not pick up any between-worker variance, while the DREAM method detected some between-worker variance.

In conclusion, for grouping dermal exposure levels of workers for our epidemiological study, both VITAE and the DREAM method could be used, obtaining the same grouping schemes. However, in this particular case the observational semi-quantitative DREAM method appeared to be more efficient, considering that the observational semi-quantitative DREAM method is easier to apply and cheaper than the VITAE method.

\section{ACKNOWLEDGEMENTS}

The authors are grateful to Erik Hagelaar, Mark Lurvink, and Martijn Kerkman for performing field measurements. We thank Roel Engel for analysing the source samples on Tinopal concentration. We would like to thank the employers and employees for participating in this study. Financial support by the Dutch Ministry of Social Affairs and Employment for this research project is gratefully acknowledged.

\section{Authors' affiliations \\ B van Wendel de Joode, Risk Assessment in the Work Environment, a collaborative centre between TNO Quality of Life and IRAS, Netherlands J Spithoven, H Kromhout, Environmental and Occupational Health Division, Institute for Risk Assessment Sciences (IRAS), Utrecht University, Utrecht, Netherlands}

D H Brouwer, TNO Quality of Life, Netherlands

E P B Bierman, TNO Human Factors, Netherlands

Competing interests: none declared

\section{REFERENCES}

1 Simpson AT, Groves JA, Unwin J, et al. Mineral oil metal working fluids (MWFs)-development of practical criteria for mist sampling. Ann Occup Hyg 2000;44:165-72.

2 National Institute for Occupational Safety and Health (NIOSH). What you need to know about occupational exposure to metal working fluids, DHHS [NIOSH] Publication 98-116. Cincinnati, OH: NIOSH, 1998.

3 Eisen EA, Bardin J, Gore R, et al. Exposure-response models based on extended follow-up of a cohort mortality study in the automobile industry. Scand J Work Environ Health 2001;27:240-9.

4 Sullivan P, Eisen E, Kriebel D, et al. A nested case-control study of stomach cancer mortality among automobile machinists exposed to metalworking fluid. Ann Epidemiol 2000;10:480-1.

5 Silverstein M, Park R, Marmor M, et al. Mortality among bearing plant workers exposed to metalworking fluids and abrasives. J Occup Med 1988;30:706-14.

6 Eisen EA, Smith TJ, Kriebel D, et al. Respiratory health of automobile workers and exposures to metal-working fluid aerosols: lung spirometry. Am J Ind Med 2001;39:443-53.

7 Greaves IA, Eisen EA, Smith TJ, et al. Respiratory health of automobile workers exposed to metal-working fluid aerosols: respiratory symptoms. Am J Ind Med 1997;32:450-9.

8 Hodgson MJ, Bracker A, Yang C, et al. Hypersensitivity pneumonitis in a metal-working environment. Am J Ind Med 2001;39:616-28.

9 de Boer EM, van Ketel WG, Bruynzeel DP. Dermatoses in metal workers (I) iritant contact dermatitis. Contact Dermatitis 1989;20:212-18.

10 de Boer EM, van Ketel WG, Bruynzeel DP. Dermatoses in metal workers (II). Allergic contact dermatitis. Contact Dermatitis 1989;20:280-6.

11 Piacitelli GM, Sieber WK, O'Brien DM, et al. Metalworking fluid exposures in small machine shops: an overview. AlHAJ 2001;62:356-70. 
12 O'Brien DM, Piacitelli GM, Sieber WK, et al. An evaluation of short-term exposures to metalworking fluids in small machine shops. AlHAJ 2001;62:342-8.

13 Woskie SR, Virii MA, Kriebel D, et al. Exposure assessment for a field investigation of the acute respiratory effects of metalworking fluids. I. Summary of findings. Am Ind Hyg Assoc J 1996;57:1 154-62.

14 Abrams L, Seixas N, Robins T, et al. Characterization of metalworking fluid exposure indices for a study of acute respiratory effects. Appl Occup Environ Hyg 2000;15:492-502.

15 Wassenius $\mathrm{O}$, Jarvholm B, Engstrom T, et al. Variability in the skin exposure of machine operators exposed to cutting fluids. Scand J Work Environ Health 1998;24:125-9.

16 Sprince NL, Palmer JA, Popendorf W, et al. Dermatitis among automobile production machine operators exposed to metal-working fluids. Am J Ind Med 1996;30:421-9.

17 Roff M, Bagon DA, Chambers H, et al. Dermal exposure to electroplating fluids and metalworking fluids in the UK. Ann Occup Hyg 2004;48:209-17.

18 Vermeulen R, Stewart P, Kromhout H. Dermal exposure assessment in occupational epidemiologic research. Scand J Work Environ Health 2002;28:371-85.

19 Soutar A, Semple S, Aitken RJ, et al. Use of patches and whole body sampling for the assessment of dermal exposure. Ann Occup Hyg 2000;44:51 1-18.

20 Cherrie JW, Brouwer DH, Roff $M$, et al. Use of qualitative and quantitative fluorescence techniques to assess dermal exposure. Ann Occup Hyg 2000;44:519-22.

21 Van Wendel de Joode B, Brouwer DH, Vermeulen R, et al. DREAM: a method for semi-quantitative dermal exposure assessment. Ann Occup Hyg 2003;47:71-87.
22 Van Wendel de Joode B, van Hemmen JJ, Meijster T, et al. Reliability of a semi-quantitative method for dermal exposure assessment (DREAM). J Expo Anal Environ Epidemiol 2005; 15:111-20.

23 Van Wendel de Joode B, Vermeulen R, van Hemmen JJ. Accuracy of a semi quantitative method for Dermal Exposure Assessment (DREAM). Occup Environ Med 2005;62:623-32.

24 Van Wendel de Joode B, Vermeulen R, Heederik D, et al. Evaluation of two self-administered questionnaires to ascertain dermatitis among metal workers and its relation with exposure to metal working fluids. Submitted.

25 Fenske RA, Leffingwell JT, Spear RC. A video imaging technique for assessing dermal exposure. I. Instrument design and testing. Am Ind Hyg Assoc J 1986;47:764-70.

26 Fenske RA, Wong SM, Leffingwell JT, et al. A video imaging technique for assessing dermal exposure. II. Fluorescent tracer testing. Am Ind Hyg Assoc J 1986;47:771-5.

27 Bierman EPB, Brouwer DH, van Hemmen JJ. Implementation and evaluation of the fluorescent tracer technique in greenhouse exposure studies. Ann Occup Hyg 1998;42:467-75.

28 Conway EJ. Microdiffusion analysis and volumetric error, 4th edn. London: Crosby Lockwood and Son, 1957.

29 Prais SJ, Aitchinson J. The grouping of observations in regression analysis. Rev Int Stat Inst 1954;1(3): 1-22.

30 Kromhout H, Heederik D. Occupational epidemiology in the rubber industry: implications of exposure variability. Am J Ind Med 1995;27:171-85

31 Kromhout H, Vermeulen R. Temporal, personal and spatial variability in dermal exposure. Ann Occup Hyg 2001;45:257-73.

\section{Call for papers}

11 th European Forum on Quality Improvement in Health Care 26-28 April 2006, Prague, Czech Republic Deadline 30 September 2005.

For further information and to submit online go to: www.quality.bmipg.com 